08

\title{
Управление температурными полями в процессе искрового плазменного спекания термоэлектриков
}

\author{
( Л.П. Булат, ${ }^{1}$ А.В. Новотельнова, ${ }^{1,9}$ А.С. Тукмакова, ${ }^{1}$ Д.Е. Ережеп, ${ }^{1}$ В.Б. Освенский, ${ }^{2}$ \\ А.И. Сорокин, ${ }^{2}$ Д.А. Пшенай-Северин, ${ }^{3}$ С. Ашмонтас ${ }^{4}$ \\ ${ }^{1}$ Университет ИТМО, \\ 197101 Санкт-Петербург, Россия \\ ${ }^{2}$ Государственный научный центр АО „Гиредмет“, \\ 119017 Москва, Россия \\ ${ }^{3}$ Физико-технический институт им. А.Ф. Иофрфе РАН, \\ 194021 Санкт-Петербург, Россия \\ ${ }^{4}$ State Research Institute Center for Physical Science and Technology, \\ LT-10222 Vilnius, Lithuania \\ ฯ e-mail: novotelnova@yandex.ru
}

(Поступило в Редакцию 30 августа 2016 г.)

Выполнено моделирование процесса создания термоэлектриков методом искрового плазменного спекания наноструктурированных порошков для получения материалов с улучшенными термоэлектрическими свойствами. Проанализированы факторы, влияющие на распределение теплового поля в процессе спекания. Рассмотрено влияние геометрических параметров оснастки на формирование температурного градиентного поля, необходимого для эффективного спекания функционально-градиентных материалов и составных ветвей термоэлементов. Результаты работы могут использоваться для определения условий и режимов спекания функционально-градиентных материалов на установках искрового плазменного спекания и горячего прессования.

DOI: $10.21883 /$ JTF.2017.04.44320.2025

\section{Введение}

Получение энергии традиционными методами, основанными на сжигании органического топлива, вызывает истощение энергетических ресурсов и нарастание экологических проблем, связанных с выбросами $\mathrm{CO}_{2}$, парниковым эффектом и тепловым загрязнением окружающей среды. Одним из способов минимизации негативного воздействия на окружающую среду является использование экологически чистых методов прямого преобразования энергии для утилизации низкопотенциального тепла. Так, с помощью термоэлектрических генераторов можно проводить утилизацию отработанного тепла от агрегатов транспортных средств, электростанций и промышленных установок, утилизировать тепло в возобновляемых источниках энергии (гибридные фототермоэлектрические солнечные батареи) и пр. [1].

Широкое распространение термогенерирующих устройств для различных приложений сдерживает их низкая эффективность. Эффективность работы термоэлектрических устройств в значительной мере определяется свойствами используемых в них термоэлектрических материалов (термоэлектриков). Для оценки свойств термоэлектриков используют параметр термоэлектрической добротности

$$
Z=\frac{\sigma \alpha^{2}}{\kappa},
$$

где $\sigma, \alpha$ и $\kappa-$ коэффициенты электропроводности, термо ЭДС и теплопроводности термоэлектрика, зависящие от температуры $T$. В настоящее время безразмерная добротность $Z T$ коммерчески доступных термоэлектрических материалов не превышает единицу.

Использование нанотехнологий может существенно увеличить ZT [2-4]. В последнее десятилетие были опубликованы работы о принципиальных достижениях в разработке высокоэффективных наноструктурированных термоэлектриков. Так, например, было заявлено, что в наноструктурированных материалах на основе соединений $\mathrm{Bi}-\mathrm{Sb}-\mathrm{Te}$ получены значения $Z T=1.4$ при $T=373 \mathrm{~K}$ и $Z T=1.2$ при комнатной температуpe $[5,6]$. Увеличение термоэлектрической добротности наноструктурированных термоэлектриков может быть связано с туннелированием носителей через зазор между нанозернами, дополнительным рассеянием фононов на границах нанозерен и энергетической фильтрацией носителей через барьеры [7-9]. Наноструктурированные порошковые материалы могут быть получены методами механоактивации с использованием шаровых мельниц, коллоидного синтеза и пр. Дальнейшая их обработка включает компактирование под давлением. Тепловое и механическое воздействие на материал в процессе компактирования может существенным образом влиять на его свойства. Например, использование горячего прессования нанопорошка способно приводить к росту зерна и частичной или полной потере достигнутого улучшения свойств.

Сохранение размера зерен в процессе термообработки возможно при использовании искрового плазменного 
спекания (ИПС) (spark plasma sintering - SPS). Tермическое воздействие на материал в процессе ИПС производится в вакууме импульсами постоянного тока при одновременном одноосном приложении давления 30-50 MPa [10-12]. Длительность процесса ИПС и достигаемая при этом температура в объеме образца должны быть достаточными для достижения механической прочности спеченного компакта. Такой способ обработки позволяет предотвратить процесс рекристаллизации и сохранить наноструктурные размеры частиц, входящих в состав компакта $[3,4]$.

Другим путем повышения эффективности термоэлементов может быть оптимизация состава материала его ветвей. В процессе эксплуатации температура термоэлемента изменяется от значений температуры „горячего“ спая до температуры „холодного“ спая. При использовании однородного термоэлектрика термоэлектрическая добротность в процессе эксплуатации будет меняться вдоль ветви, так как она зависит от температуры. Для оптимизации величины добротности возможно использование термоэлектриков с равномерно изменяющимся по длине ветви составом или составных ветвей [13].

У функционально-градиентных термоэлектриков и/или составных ветвей теплофизические параметры, такие как тепло- и электропроводность, теплоемкость, термо ЭДС, изменяют свое значение в объеме материала вдоль выделенного направления. При этом температура спекания также должна изменяться вдоль этого направления. Материалы, эксплуатация которых происходит при больших температурах, требуют, как правило, и термообработки в процессе спекания при больших температурах. То есть при получении функциональноградиентных материалов или составных ветвей термоэлементов необходимо наличие температурного перепада во время создания ветви, так как каждый слой будет иметь свою оптимальную температуру спекания. Температура каждого слоя должна быть достаточной для успешного спекания и формирования структуры будущего материала. В то же время нельзя допускать перегрева, так как он приведет к рекристаллизации, росту зерна и, как следствие, к потере качества изделия.

Обработка неоднородного по составу термоэлектрика или создание при спекании составной ветви термоэлемента предполагает обработку образца в процессе ИПС в градиентном температурном поле.

Таким образом, задача повышения эффективности термоэлектрических материалов путем создания объемных наноструктур с контролируемыми свойствами является актуальной проблемой.

В настоящей работе рассмотрено получение объемных наноструктурированных материалов для ветвей термоэлементов при обработке в градиентном температурном поле в процессе ИПС. Методом конечных элементов произведено моделирование распределения температурного поля в объеме образца при модификации геометрических параметров элементов, входящих в состав оснастки установки ИПС. Асимметричность формы матрицы и ее расположения могут влиять на конфигурацию температурного поля в процессе спекания [14]. Изменяя геометрические параметры оснастки: высоты пуансонов, расположения матрицы пресс-формы, формы и размеров ее основания, можно формировать градиентное температурное поле в образце. В качестве исследуемого материала выбраны твердые растворы на основе халькогенидов висмута, как наиболее эффективные и широко применяемые термоэлектрики $[15,16]$, а также теллурид свинца [17] и твердые растворы силицида и станнида магния [18].

\section{1. Используемая модель}

Моделирование искрового плазменного спекания осуществлялось на примере спекания в установке SPS-511S образцов теллурида висмута $\mathrm{Bi}_{2} \mathrm{Te}_{3}$, теллурида свинца $\mathrm{PbTe}$, твердого раствора станнида и силицида магния $\mathrm{Mg}_{2} \mathrm{Si}_{0.4} \mathrm{Sn}_{0.6}$ и составных образцов, содержащих слои одинаковой толщины перечисленных термоэлектриков [19]. В [20] был рассмотрен процесс ИПС таких образцов в равномерном и симметричном относительно центра образца температурном поле. Было показано, что как локальная, так и объемная неоднородности состава термоэлектрика и его теплофизических свойств существенно не влияют на распределение температурного поля в образце в ИПС процессе. Методика задания свойств материала образца и деталей установки приведена в [16].

Схематическое представление установки приведено на рис. 1. Модель включает в себя токоведущие части (стальные электроды, графитовые вставки, пуансоны, матрицу пресс-формы и образец) и электрически изолированный корпус вакуумной камеры.

В процессе искрового плазменного спекания через верхний и нижний охлаждаемые водой электроды (1 и 8, рис. 1) пропускается импульсный ток. Длительность импульса составляет $3.3 \mathrm{~ms}$, скважность 2.2; за каждым пакетом из 12 импульсов следует 2 периода отключения тока.

Амплитуда импульсов изменяется в процессе спекания, благодаря чему изменяется эффективное значение плотности тока. В начале процесса происходит увеличение эффективного значения тока по закону, близкому к линейному. При этом в токопроводящих частях установки и образце происходит увеличение температуры. Рост температуры спекаемого образца происходит вследствие джоулева разогрева. При рассматриваемых частотах скин-эффектом можно пренебречь и учитывать для спекания проводящих сред только разогрев Джоуля [21]. Динамика изменения температуры коррелирует с динамикой изменения тока. По достижении заданного значения температуры происходит термостатирование образца (выдержка в течение 10-30 min при постоянной температуре). Основное термическое воздействие на образец происходит в этот временной промежуток. Эффективное значение тока при этом практически не 


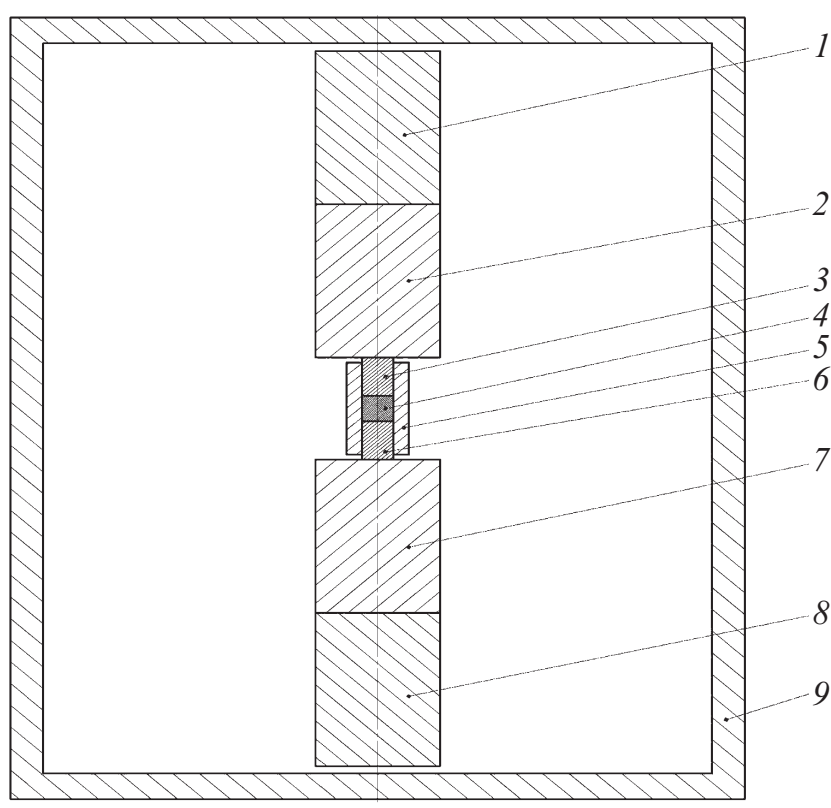

Рис. 1. Схематическое представление установки искрового плазменного спекания: 1 - верхний охлаждаемый электрод $\left(d_{e}=80 \mathrm{~mm}, h_{e}=60 \mathrm{~mm}\right) ; 2,7$ - графитовые вставки $\left(d_{g}=80 \mathrm{~mm}, h_{g}=60 \mathrm{~mm}\right) ; 3-$ верхний графитовый пуансон $\left(d_{p}=20 \mathrm{~mm}, h_{p t}=20 \mathrm{~mm}\right) ; 6-$ нижний графитовый пуансон $\left(d_{p}=20 \mathrm{~mm}, h_{p b}=20 \mathrm{~mm}\right) ; 4$ - образец $\left(d_{s}=20 \mathrm{~mm}\right.$, $\left.h_{s}=21 \mathrm{~mm}\right) ; 5-$ матрица пресс-формы $\left(d_{d . \mathrm{int}}=20 \mathrm{~mm}\right.$, $\left.d_{d . \mathrm{ext}}=40 \mathrm{~mm}, h_{d}=40 \mathrm{~mm}\right) ; 8-$ нижний охлаждаемый электрод $\left(d_{e}=80 \mathrm{~mm}, h_{e}=60 \mathrm{~mm}\right) ; 9-$ корпус вакуумной камеры.

изменяется. Поэтому задачу исследования распределения тепловых и электрических полей можно решать как стационарную, заменяя импульсный ток на его эффективное значение.

Моделирование выполнено методом конечных элементов в программном пакете COMSOL Multiphysics. Основными уравнениями задачи являются законы сохранения заряда

$$
\operatorname{divj}=0 \text {, }
$$

энергии

$$
c_{p} \rho \frac{\partial T}{\partial t}+\operatorname{divq}+\mathbf{j} \nabla \varphi=0
$$

и феноменологические законы Ома и Фурье для плотностей тока $\mathbf{j}$ и потока тепла $\mathbf{q}$

$$
\mathbf{j}=-\sigma \nabla \varphi, \quad \mathbf{q}=-\kappa \nabla T
$$

Здесь $\varphi-$ электрохимический потенциал, $\sigma$ и $\kappa-$ соответственно коэффициенты электро- и теплопроводности, $c_{p}$ и $\rho-$ удельная теплоемкость при постоянном давлении и плотность материала, $\nabla T-$ градиент температуры.

\section{2. Граничные условия}

В модели приняты следующие граничные условия для тепловых величин:

1. Внешняя поверхность камеры имеет температуру, равную температуре окружающей среды $(300 \mathrm{~K})$.

2. На нижнем и верхнем электродах для описания водяного охлаждения использовались условия конвективного теплообмена

$$
q_{c o n v}=K_{b}\left(T-T_{0}\right),
$$

где для контакта стали с водой был выбран коэффициент $K_{b}=370 \mathrm{~W} /\left(\mathrm{m}^{2} \mathrm{~K}\right)$.

3. Между внешней поверхностью нагревающихся токоведущих частей установки и внутренней поверхностью изолированного корпуса камеры осуществлялся радиационный теплообмен [22,23]. Плотность потока излучения реального тела для поверхностей внутри камеры $J, \mathrm{~W} / \mathrm{m}^{2}$

$$
\begin{aligned}
J= & (1-\varepsilon) G+\varepsilon e_{b}(T)=(1-\varepsilon) \\
& \times\left(G_{m}(J)+G_{\mathrm{ext}}+G_{\mathrm{amb}}\right)+\varepsilon e_{b}(T) .
\end{aligned}
$$

Здесь $(1-\varepsilon) G$ - плотность отраженного лучистого потока, $\varepsilon-$ коэффициент черноты реального тела (параметры $\varepsilon$ для графита и стали были приняты равными 0.75 и 0.675 соответственно), $\varepsilon e_{b}(T)-$ плотность лучистого потока, испускаемого телом, $e_{b}(T), \mathrm{W} / \mathrm{m}^{2}-$ плотность потока излучения абсолютно черного тела, равная

$$
e_{b}(T)=\sigma T^{4}
$$

где $\sigma=5.67 \cdot 10^{-8} \mathrm{~W} /\left(\mathrm{m}^{2} \cdot \mathrm{K}^{4}\right)$ - постоянная Стефана-Больцмана, $T$ - абсолютная температура.

$G$ - плотность потока лучистой энергии, падающего на поверхности установки, $\mathrm{W} / \mathrm{m}^{2}$

$$
G=G_{m}+G_{\mathrm{ext}}+G_{\mathrm{amb}},
$$

где $G_{m}-$ плотность лучистого потока от поверхности установки, $G_{\text {ext }}$ - плотность лучистого потока от внешних источников, $G_{\text {amb }}$ - плотность лучистого потока, исходящего от окружающей среды

$$
G_{\mathrm{amb}}=F_{\mathrm{amb}} e_{b}\left(T_{\mathrm{amb}}\right),
$$

где $F_{\mathrm{amb}}$ - угловой коэффициент, зависящий от площади и взаимного расположения поверхностей.

Суммарная плотность теплового потока с учетом затрат на радиацию $\mathrm{W} / \mathrm{m}^{2}$ описывается при помощи закона Фурье в векторной форме

$$
-\mathbf{n}(-\kappa \Delta T)=\varepsilon\left(G-e_{b}(T)\right),
$$

где $\mathbf{n}-$ вектор нормали, $\kappa-$ коэффициент теплопроводности, $\mathrm{W} / \mathrm{m} \cdot \mathrm{K}$.

4. Для описания электрических полей в граничных условиях на верхнем электроде задавалось значение 

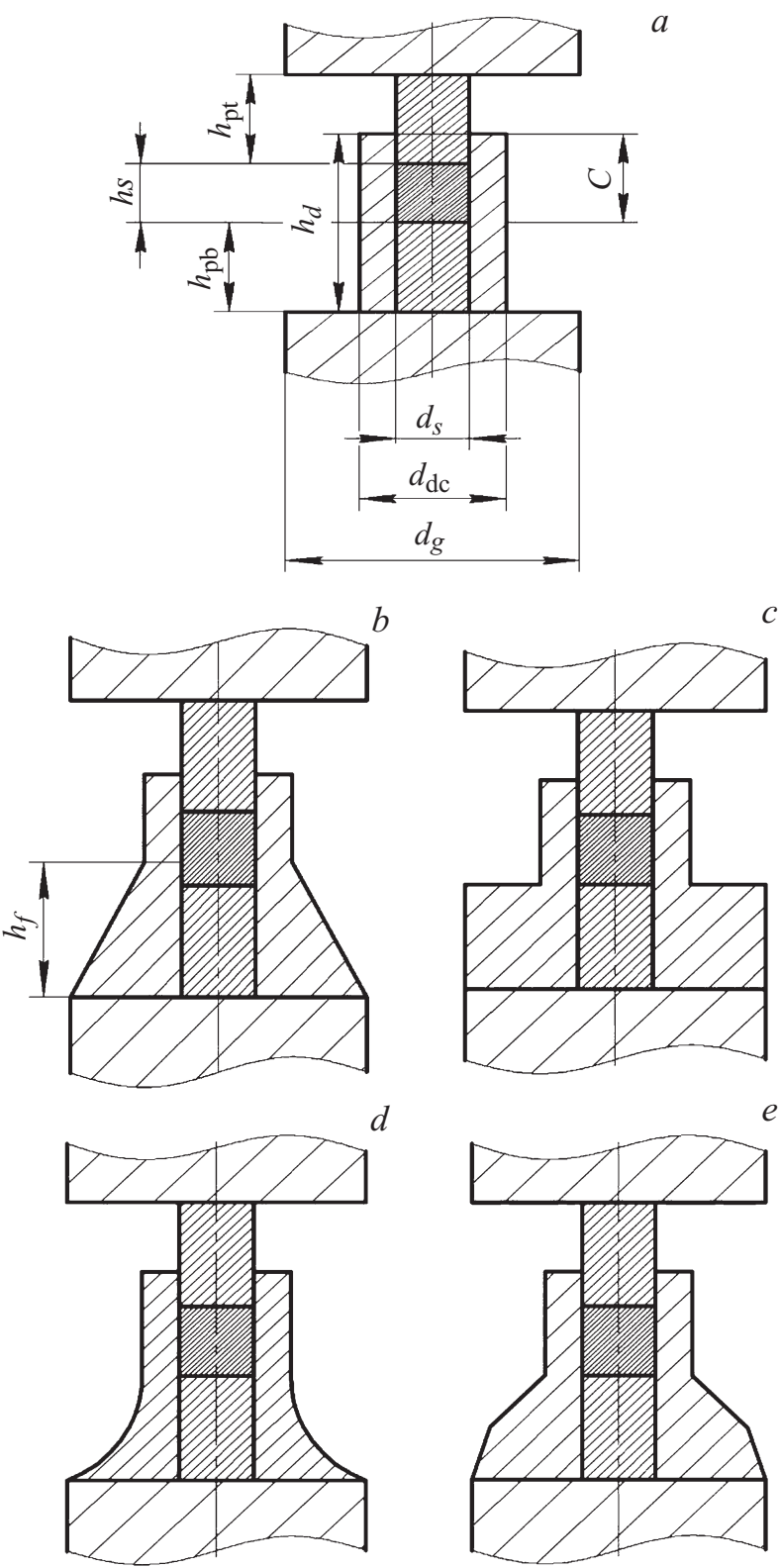

Рис. 2. Схематичное представление фрагмента установки искрового плазменного спекания с матрицей пресс-формы различной геометрии основания: $a-$ цилиндрическая матрица без расширения основания; $b-\mathrm{c}$ коническим профилем; $c-$ со ступенчатым профилем; $d-$ с вогнутым профилем; $e-\mathrm{c}$ выгнутым профилем.

плотности постоянного тока, соответствующее эффективному значению импульсного тока, достаточного для достижения на нижней границе образца температуры, достаточной для спекания термоэлектрика.

Для сохранения наноразмерных размеров зерен, входящих в состав компакта, температуры спекания не должны превышать 70-80\% от температуры плавления. Для исследованных термоэлектриков значения температур спекания составили $730 \mathrm{~K}$ для теллурида висмута, 770 К для теллурида свинца и $910 \mathrm{~K}$ для твердого раствора станнида магния.
Нижнему стальному электроду приписывался потенциал, равный потенциалу земли.

\section{3. Основные результаты}

В процессе моделирования нас интересовало распределение температурных полей в установке и образце. Распределение температурного поля внутри установки и в образце проанализировано при различных конфигурациях расположения и геометрических размерах оснастки и положения образца в ней.

\section{1. Слабая неоднородность температурного поля при симметричном расположении матрицы цилиндрической формы}

Для получения образцов, однородных по составу и свойствам, термообработка в ИПС процессе производится в установке с симметричным расположением цилиндрической матрицы пресс-формы относительно образца (рис. 1). В этом случае тепловое поле в образце распределено практически равномерно. Максимальных значений температура достигает в центре образца $[16,20]$. Перепад температур между центром образца и его поверхностью составляет единицы градусов.

Тепловой поток распространяется от центра образца к его периферии и проходит через элементы установки в направлении охлаждаемых электродов. Область, в которой тепловой поток меняет свое направление, расположена в центре образца. Распределение теплового потока зеркально симметрично. Температура верхней и нижней сторон образца в этом случае при спекании будет одинакова (рис. 3, кривая 1 ).

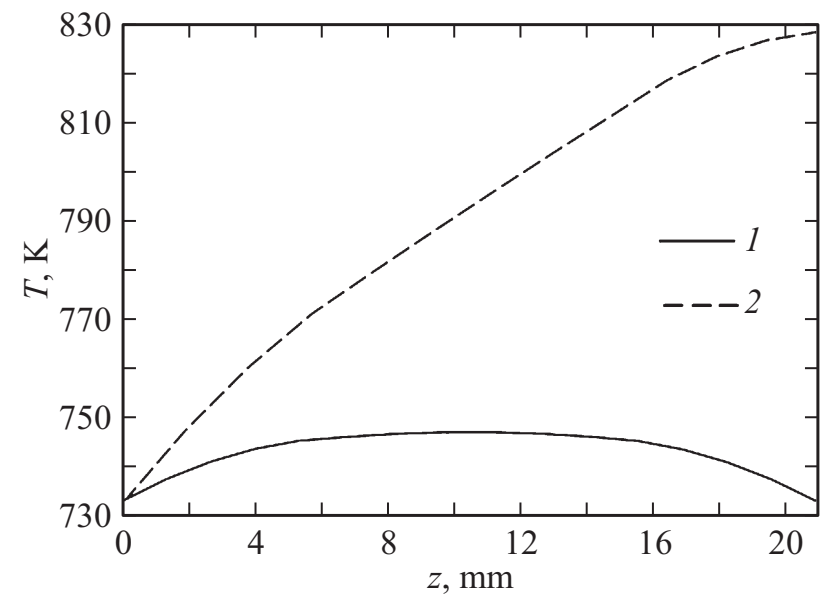

Рис. 3. Распределение температуры вдоль оси $z$ образца $\left(d_{s}=20 \mathrm{~mm}, h_{s}=21 \mathrm{~mm}\right): 1-$ цилиндрическая матрица, расположенная симметрично относительно образца, 2 - цилиндрическая матрица, контактирующая с нижней графитовой вставкой. 


\section{2. Влияние расположения матрицы пресс-формы на формирование температурного поля}

Для формирования градиентного температурного поля в образце вдоль его вертикальной оси можно изменять условия теплопередачи на внутренних границах образец - графит (графитовая матрица, пуансоны), смещая матрицу вниз так, чтобы образовался контакт между матрицей и нижней графитовой вставкой (рис. 2,a). С одной стороны, появление этого контакта нарушает геометрическую симметричность верхней и нижней половин установки. С другой, этот контакт способствует усилению теплоотвода от поверхности образца посредством теплопроводности через нижнюю графитовую вставку к охлаждаемому электроду. Определяющую роль в появлении градиентного температурного поля играет наличие электрического и теплового контактов нижнего торца матрицы пресс-формы с графитовой вставкой $[14,24]$. Появление контакта нижней границы матрицы с графитовой вставкой вызывает появление перепада температур $\Delta T$ между верхней и нижней границами образца (рис. 3, кривая 2).

\section{3. Влияние положения образца в оснастке установки на формирование градиентного температурного поля}

Увеличить градиент температуры в образце можно путем смещения его положения в цилиндрической матрице пресс-формы относительно центра установки. При моделировании этой ситуации изменялась высота нижнего пуансона $h_{p b}$. Высота верхнего пуансона оставалась неизменной. Положение образца в матрице описывалось параметром $c=h_{d}-h_{p b}$, соответствующим расстоянию между нижней поверхностью образца и верхней границей матрицы при фиксированной высоте образца (рис. 2,a).

На рис. 4 приведена зависимость перепада температур $\Delta T$ от положения образца в матрице пресс-формы для образцов различной высоты $h_{s}$. Смещение образца к нижней границе матрицы пресс-формы приводит к увеличению $\Delta T$.

\section{4. Влияние конфигурации основания матрицы пресс-формы на формирование температурного поля}

Изменению условий теплопроводности также может способствовать увеличение площади контакта между матрицей и нижней графитовой вставкой. Увеличение диаметра цилиндрической матрицы существенного вклада в асимметрию установки и рост перепада температур не внесет. Поэтому для увеличения асимметрии была изменена форма матрицы. Нижняя часть была расширена за счет появления основания. Верхняя часть матрицы была оставлена неизменной в форме полого цилиндра с внешним диаметром $d_{d c}$.

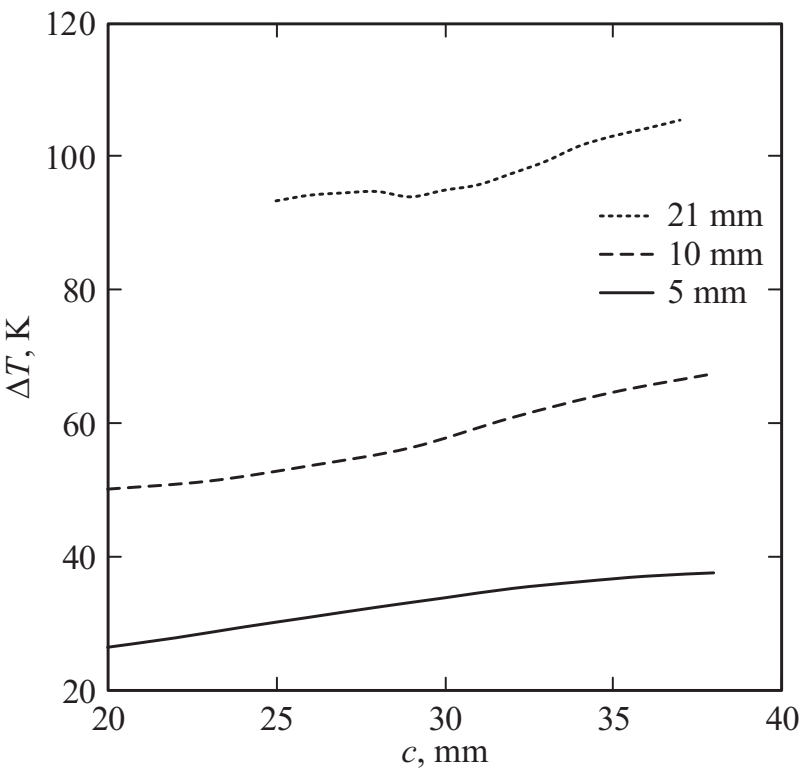

Рис. 4. Зависимость перепада температур от положения образца в матрице для различной высоты образца $h_{s} 5,10$ и $21 \mathrm{~mm}$.

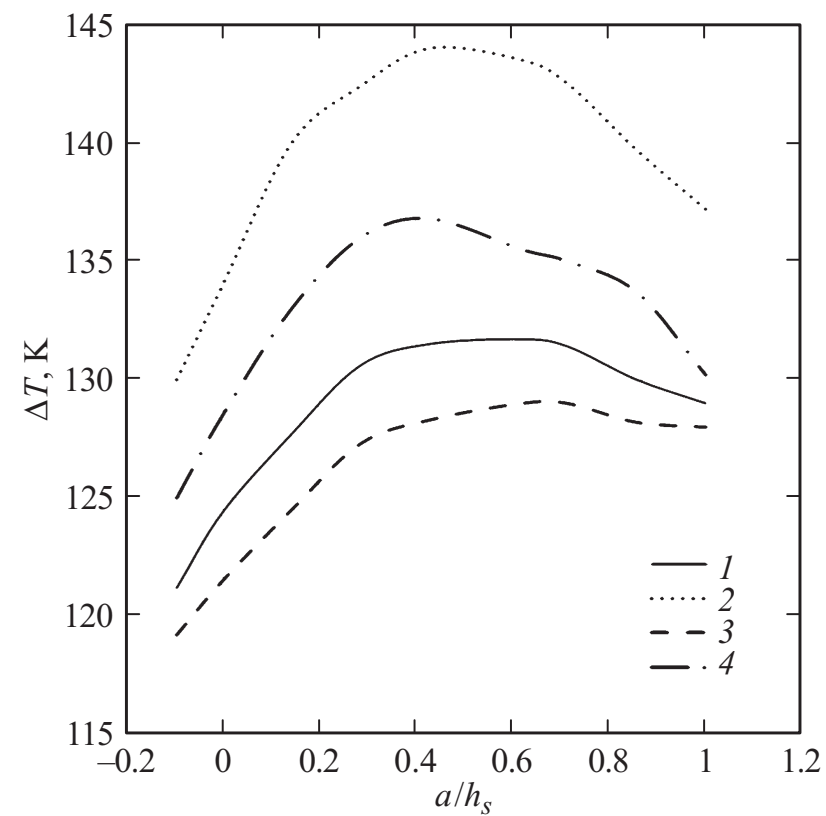

Рис. 5. Зависимость перепада температур во время спекания от отношения $a / h_{s}$ для различных геометрических форм основания матрицы пресс-формы: 1 - конический профиль; 2 - ступенчатый профиль; 3 - вогнутый профиль; 4 выпуклый профиль.

На характер и интенсивность теплоотвода может влиять форма основания матрицы. Для анализа влияния геометрической формы основания на температурное поле в образце было проведено моделирование процесса спекания с матрицами пресс-формы с измененным профилем. Были рассмотрены следующие конфигурации 
основания матрицы пресс-формы: коническая (рис. 2, $b$ ), ступенчатая (рис. 2,c), с вогнутой и выпуклой поверхностями (рис. 2, $d, e$ ).

Принятые на рис. 2 обозначения: $h_{d}$ - высота матрицы пресс-формы, $h_{f}$ - высота основания матрицы, $h_{p t}$ и $h_{p b}$ - высота верхнего и нижнего пуансонов соответственно, $d_{s}$ - диаметр образца, $d_{d c}$ - диаметр верхней цилиндрической части матрицы, $d_{g}$ - диаметр нижней графитовой вставки.

Наружный диаметр нижней части основания матрицы пресс-формы был выбран равным диаметру нижней графитовой вставки $d_{g}$ для обеспечения лучших условий теплообмена между этими деталями.

Положение наружной части матрицы относительно низа образца может быть охарактеризовано параметром

$$
a=h_{f}-h_{p b}
$$

На рис. 5 приведена зависимость перепада температур $\Delta T$ между верхней и нижней сторонами образца теллурида висмута высотой $21 \mathrm{~mm}$ (высота пуансонов составляла $15 \mathrm{~mm}$ ) при спекании с использованием матриц пресс-формы различной геометрии. Для всех использованных в анализе форм матриц пресс-формы прослеживается корреляция перепада температур со степенью перекрытия образца основанием матрицы прессформы $a / h_{s}$. Наибольшего значения перепада температур удается достичь при использовании ступенчатого профиля матрицы пресс-формы.

\section{5. Влияние условий теплоотвода с поверхности установки}

Между внешней поверхностью матрицы пресс-формы и поверхностями вакуумной камеры и элементами установки осуществляется радиационный теплообмен, однако его вкладом в формирование температурного поля внутри образца можно пренебречь. Это подтверждается сопоставлением результатов распределения температурных полей, полученных ранее в работах $[14,16,20]$, с результатами моделирования процесса спекания с использованием граничными условий, принятых в настоящей работе. Внесение теплоизоляции внешней поверхности матрицы пресс-формы также не дало видимого изменения в распределении температурного поля в образце и оснастке. Изменение граничных условий по теплу на внешней поверхности матрицы не вносит существенного вклада в формирование перепада температур по высоте образца. Это связано, по-видимому, с недостаточно высокой температурой внешней поверхности частей установки, которая могла бы интенсифицировать радиационный теплообмен. Также это может быть объяснено значительным расстоянием между нагретыми частями установки и внутренней поверхностью стенок вакуумной камеры.

\section{4. Обсуждение результатов}

Оснастка установки (графитовые матрица прессформы и пуансоны) в процессе ИПС не только определяет геометрию образца при прессовании, но и активно участвует в формировании тепловых и температурных полей. Протекающий в процессе ИПС ток производит нагрев образца и элементов оснастки. Тепловой поток направлен от центра установки к электродам, охлаждаемым водой. Выделяемая теплота посредством теплопроводности перераспределяется между образцом, пуансонами и матрицей. На интенсивность процесса теплоотвода можно влиять, изменяя конфигурацию оснастки (высоту, площадь сечения и форму ее элементов) за счет изменения теплового сопротивления участка тепловой цепи $R_{t}$ :

$$
R_{t}=\frac{l}{\kappa S}
$$

где $l$ - длина участка тепловой цепи, $S-$ площадь его сечения.

Изменение конфигурации оснастки приводит к изменению выделения теплоты Джоуля. При использовании симметричной оснастки области с максимальным выделением теплоты Джоуля располагаются у верхней границы верхнего пуансона и нижней границы нижнего пуансона. Тепловое и электрическое сопротивления верхней и нижней частей оснастки распределены зеркально-симметрично относительно горизонтальной оси. Максимальных значений температура в этом случае достигает в центральной области образца.

При смещении матрицы к нижней графитовой вставке область с наибольшим выделением джоулевой теплоты располагается в верхнем пуансоне, что приводит к большему разогреву верхней части оснастки. Создание контакта между матрицей и нижней графитовой вставкой приводит к снижению теплового сопротивления нижней части оснастки, вызванному увеличением площади поперечного сечения оснастки у нижней границы пуансона.

Дополнительному снижению теплового сопротивления нижней части матрицы способствует введение в конструкцию матрицы пресс-формы расширенного основания. Ступенчатый профиль основания матрицы прессформы по сравнению с другими конфигурациями обладает меньшим тепловым сопротивлением и обеспечивает более интенсивный отвод теплоты в направлении нижнего электрода.

Тепловые процессы, протекающие в образце и установке, можно описать при помощи мощности теплового потока, проходящего через верхнюю поверхность образца $Q_{t}$ и нижнюю поверхность $Q_{b}$. Мощность теплового потока, проходящего через поверхности образца, формируется за счет джоулевой теплоты, выделяемой при прохождении электрического тока. При постоянном значении плотности тока $Q$ зависит от теплофизических свойств материалов токопроводящих частей установки и их геометрических размеров. 
Изменяя площадь поперечного сечения матрицы пресс-формы, мы изменяем плотность теплового потока $q, \mathrm{~W} / \mathrm{m}^{2}$

$$
q=\frac{d Q}{d S}
$$

где $S$ - площадь сечения, через которую проходит тепловой поток, $\mathrm{m}^{2}$.

В случае симметрично расположенной матрицы пресс-формы мощности тепловых потоков через верхнюю и нижнюю поверхности образца равны по абсолютной величине. Так, для образца высотой $21 \mathrm{~mm}$ и диаметром $20 \mathrm{~mm}$ они составят $Q_{t}=8.03 \mathrm{~W}$ и $Q_{b}=8.03 \mathrm{~W}$. То есть тепловой поток является симметричным относительно центра образца, и разница температур между нижней и верхней поверхностями образца отсутствует.

При несимметричном расположении матрицы прессформы картина распределения тепловых потоков в установке изменяется. На верхней поверхности образца тепловой поток становится положительным, что соответствует нагреву и росту температуры. На нижней поверхности тепловой поток остается отрицательным и увеличивает свое абсолютное значение, что отвечает более интенсивному отводу тепла. При увеличении разности между отведенным и подведенным тепловыми потоками увеличивается значение $\Delta T$. Данный эффект усиливается при дополнительном расширении матрицы при помощи основания.

Высота основания матрицы оказывает влияние на распределение тепловых полей. На рис. 6 приведена зависимость мощности тепловых потоков $Q_{t}$ и $Q_{b}$ для образца теллурида висмута высотой $21 \mathrm{~mm}$ и диаметром $20 \mathrm{~mm}$ от степени перекрытия образца основанием матрицы пресс-формы. При увеличении степени перекрытия происходит смещение максимального значения теплового потока к верхнему пуансону. Нагрев верхней части образца усиливается и, хоть отвод теплоты от нижней границы несколько ослабевает, разность между количеством подведенного и отведенного тепла возрастает, что в результате приводит к росту $\Delta T$ по высоте образца.

Зависимость перепада температур от степени перекрытия имеет экстремальный характер. Это характерно для всех рассмотренных конфигураций матрицы прессформы (рис. 5). Максимального значения перепад температур между верхней и нижней сторонами образца достигает при некотором оптимальном значении степени перекрытия $\left(a / h_{s}\right)_{\text {орt. }}$ Значение $\left(a / h_{s}\right)_{\text {орt }}$ изменяется при изменении высоты образца и температуры, при которой происходит спекание. Величина $\left(a / h_{s}\right)$ орt увеличивается при использовании образцов большей высоты. Увеличение используемых температур спекания также требует больших значений $\left(a / h_{s}\right)_{\text {opt. }}$ На рис. 7 представлена зависимость $\left(a / h_{s}\right)_{\text {орt }}$ от высоты образца $h_{s}$ для образцов, выполненных из различных материалов.

Для низкотемпературных и среднетемпературных материалов значения $\left(a / h_{s}\right)_{\text {opt }}$ практически совпадают. Для образцов малой высоты до $7 \mathrm{~mm}$ желательно совпадение высоты основания матрицы пресс-формы с нижней

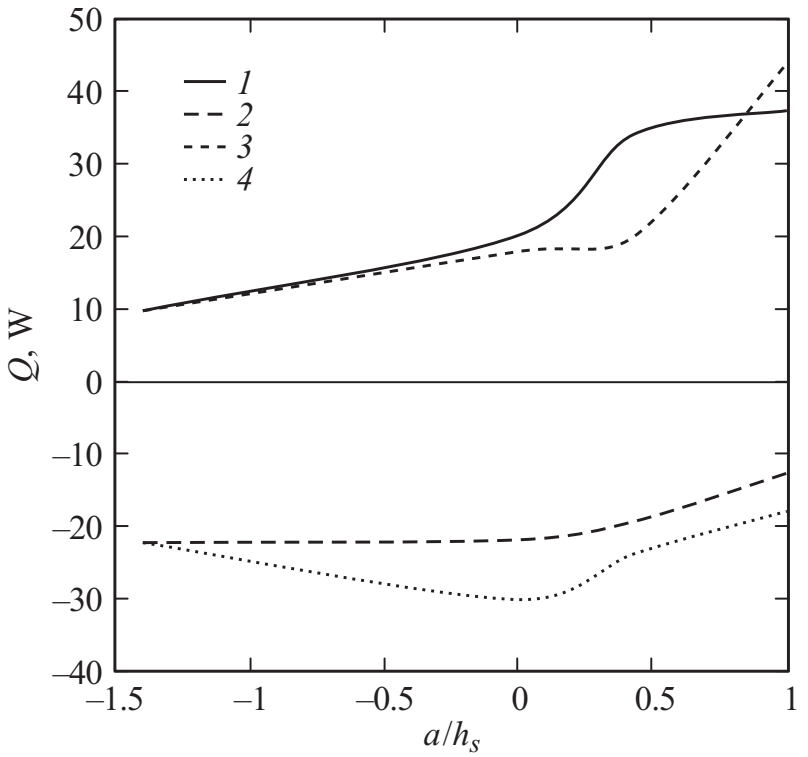

Рис. 6. Зависимость мощности теплового потока через верхнюю $Q_{t}(1,3)$ и нижнюю $Q_{b}(2,4)$ поверхности образца от $a / h_{s}$ при спекании в матрице пресс-формы с различными профилями основания: ступенчатый профиль $(1,2)$, конический профиль $(3,4)$.



Рис. 7. Зависимость степени перекрытия образца основанием матрицы, обеспечивающей максимальное значение перепада температур $\left(a / h_{s}\right)_{\text {орt }}$ от высоты образца $h_{s}$. Материал образца: $1-\mathrm{Bi}_{2} \mathrm{Te}_{3}, 2-\mathrm{PbTe}, 3-\mathrm{Mg}_{2} \mathrm{Si}_{0.4} \mathrm{Sn}_{0.6}, 4-$ составной образец.

границей образца, т. е. отсутствие перекрытия. С ростом высоты образца значение $\left(a / h_{s}\right)$ орt увеличивается до 0.5 .

Для высокотемпературных образцов высотой до $9 \mathrm{~mm}$ $\left(a / h_{s}\right)_{\text {орt }}$ изменяется в пределах $0.2-0.4$. При увеличе-

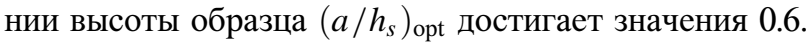


В случае составного образца высотой до $7 \mathrm{~mm}$ зависимость сходна с поведением низко- и среднетемпературных материалов. Однако с увеличением высоты максимального перепада температур удается достичь при значениях $\left(a / h_{s}\right)_{\text {opt }}$, не превышающих 0.3 .

\section{Выводы}

С помощью компьютерного моделирования процесса ИПС термоэлектрических материалов изучено влияние геометрических параметров оснастки на формирование температурного градиентного поля, необходимого для эффективного спекания функционально-градиентных материалов и составных ветвей термоэлементов

На температурное поле в образце в ИПС процессе влияет расположение образца в оснастке. Смещение образца к нижней границе матрицы пресс-формы с одновременным уменьшением высоты нижнего пуансона способствует увеличению разницы температур между нижней и верхней поверхностями образца.

Возникновение градиента температуры в спекаемом материале возможно при создании электрического контакта между матрицей прес-сформы и нижней графитовой вставкой. Его увеличению способствует изменение геометрической формы матрицы посредством введения в ее конструкцию расширенного основания

Анализ различных вариантов конфигураций основания матрицы пресс-формы показал, что лучшие результаты по созданию перепада температур по высоте образца могут быть получены для матрицы со ступенчатым профилем основания.

Распределение температуры по высоте образца зависит от степени перекрытия образца основанием матрицы пресс-формы. Найдены оптимальные значения степени перекрытия, способствующие наиболее эффективному формированию градиентного поля температур, для различных геометрических параметров и материалов образца. Предложенные в настоящей работе способы формирования градиентного поля температур позволяют создать температурный перепад по высоте образца, величина которого может достигать значений до $150 \mathrm{~K}$.

Полученные данные могут быть использованы в качестве рекомендаций по созданию составных и функционально-градиентных ветвей термоэлементов, спеченных из наноструктурированных порошковых материалов в градиентном температурном поле. Результаты работы носят универсальный характер и могут применяться на установках ИПС и установках горячего прессования, использующих режимы спекания и размеры образцов и элементов оснастки, отличные от рассмотренных в настоящей работе. Представленные результаты могут использоваться для определения условий и режимов спекания материалов, имеющих электрическую природу, отличную от исследованных в работе термоэлектрических материалов.
Работа выполнена при государственной финансовой поддержке ведущих университетов Российской Федерации (субсидия 074-U01).

\section{Список литературы}

[1] Анатычук Л.И. Термоэлектрические преобразователи энергии. Термоэлементы. Элементная база термоэлектричества. Киев: Институт термоэлектричества, 2003. 376 с.

[2] Poudel B., Hao Q., Ma Y., Lan Y., Minnich A., Yu B., Yan X., Wang D., Muto A., Vashaee D., Chen X., Liu J., Dresselhaus M.S., Chen G., Ren Z. // Science. 2008. Vol. 320. N 5876. P. 634-638.

[3] Bulat L.P., Drabkin I.A., Karatayev V.V., Osvenskii V.B., Parkhomenko Yu.N., Lavrentev M.G., Sorokin A.I., PshenaiSeverin D.A., Blank V.D., Pivovarov G.I., Bublik V.T., Tabachkova N.Yu. // J. Electron. Mater. 2013. Vol. 42. N 7. P. 2110-2113.

[4] Drabkin I.A., Osvenskii V.B., Parkhomenko Yu.N., Sorokin A.I., Pivovarov G.I., Bulat L.P. // J. Thermoelectricity. 2013. N 3. P. $35-46$.

[5] Xie W., Tang X., Yan Y., Zhang Q., Tritt M.T. // J. Appl. Phys. 2009. Vol. 105. N 11. P. 113713.

[6] Xie W., Tang X., Yan Y., Zhang Q., Tritt M.T. // Appl. Phys. Lett. 2009. Vol. 94. N 10. C. 102111.

[7] Bulat L.P., Drabkin I.A., Karatayev V.V., Osvenskii V.B., Parkhomenko Yu.N., Pshenay-Severin D.A., Sorokin A.I. // J. Electron. Mater. 2014. Vol. 43. N 6. P. 2121-2126.

[8] Bulat L.P., Bublik V.T., Drabkin I.A., Karataev V.V., Osvenskii V.B., Parkhomenko Yu.N., Pivovarov G.I., PshenaiSeverin D.A., Tabachkova N.Yu. // J. Electron. Mater. 2010. Vol. 39. N 9. P. 1650-1653.

[9] Goldsmid H.J. Introduction to thermoelectricity. Berlin: Springer-Verlag. 2016. C. 74-76.

[10] Guillon O., Gonzalez-Julian J., Dargatz B., Kessel T., Schierning G., Räthel J., Herrmann M. // Advanc. Eng. Mater. 2014. Vol. 16. N 7. P. 830-849.

[11] Драбкин И.А., Каратаев В.В., Освенский В.Б., Пархоменко Ю.Н., Сорокин А.И., Булат Л.П., Пивоваров Г.И., Бублик В.Т., Табачкова Н.Ю. // Материалы электронной техники. 2012. N 3. С. 66-69.

[12] Булат Л.П., Драбкин И.А., Новотельнова А.В., Освенский В.Б., Пшенай-Северин Д.А., Сорокин А.И., Тукмакова А.C. // Термоэлектрики и их применения. СПб.: ФТИ им. А.Ф. Иоффе, 2015. С. 40-45.

[13] Анатычук Л.И., Вихор Л.Н. // Термоэлектрики и их применения. СПб.: ФТИ им. А.Ф. Иоффе, 2013. С. 434-439.

[14] Булат Л.П., Драбкин И.А., Новотельнова А.В., Освенский В.Б., Пархоменко Ю.Н., Сорокин А.И., ПшенайСеверин Д.А., Нефедова И.А. // Письма в ЖТФ. 2014. Т. 40. № 21. C. 79-87.

[15] Scherrer H., Scherrer S. Thermoelectrics Handbook: Macro to Nano / Ed. by D.M. Rowe CRC Taylor and Francis: Boca Raton, FL, USA, 2012. 954 p.

[16] Булат Л.П., Пшенай-Северин Д.А., Нефедова И.А., Новотельнова А.В., Гуревич Ю.Г. // Научно-технический вестник информационных технологий механики и оптики. 2014. № 5 (93). С. $38-45$.

[17] Равич Ю.И., Ефимова Б.А., Смирнов И.А. Методы исследования полупроводников в применении к халькогенидам свинца PbTe, PbSe, PbS. М.: Наука, 1968. 384 с. 
[18] Zaitsev V.K., Fedorov M.I., Gurieva E.A., Eremin I.S., Konstantinov P.P., Samunin A.Yu., Vedernikov M.V. // Phys. Rev. 2006. Vol. 74. P. 045207.

[19] Bulat L.P., Novotelnova A.V., Asach A.V., Tukmakova A.S., Osvenskii V.B., Parchomenko Y.N., Zhao L., Zongrui Q. // J. Electron. Mater. 2016. Vol. 45. N 6. P. 2891-2894.

[20] Булат Л.П., Новотельнова А.В., Пиенай-Северин Д.А., Освенский В.Б., Сорокин А.И., Асач А.В., Тукмакова А.С. // ЖТФ. 2016. Т. 86. Вып. 1. С. 70-77.

[21] Anselmi-Tamburinia U., Gennarib S., Garaya J.E., Munir Z.A. // Mater. Sci. Engineer. 2005. A. 394. P. 139-148.

[22] Modest M.F. Radiative Heat Transfer, 2nd ed. Academic Press, San Diego, California, 2003. 842 p.

[23] Sieger R., Howell J. Thermal Radiation Heat Transfer, 4th ed. Taylor \& Francis, N. Y., 2002. 1072 p.

[24] Булат Л.П., Новотельнова А.В., Тукмакова А.С., Ережеп Д.Е., Опря К.A. // Научный журнал НИУ ИТМО. Сер. Холодильная техника и кондиционирование. 2015. T. 4. N 20. C. 9-17. 